\title{
Mechanism of Interaction Between Cefonicid Sodium and Trypsin by Spectroscopic and Molecular Docking Methods
}

\author{
Jinju Wang, Baosheng Liu*, Shaotong Duan, Tongtong Li \\ Key Laboratory of Analytical Science and Technology of Hebei Province, College of Chemistry \& Environmental Science, Hebei University, \\ Baoding, P. R. China
}

Email address:

lbs@hbu.edu.cn (Baosheng Liu)

${ }^{*}$ Corresponding author

\section{To cite this article:}

Jinju Wang, Baosheng Liu, Shaotong Duan, Tongtong Li. Mechanism of Interaction Between Cefonicid Sodium and Trypsin by Spectroscopic and Molecular Docking Methods. American Journal of Optics and Photonics. Vol. 5, No. 6, 2017, pp. 80-87.

doi: 10.11648/j.ajop.20170506.14

Received: January 10, 2017; Accepted: January 31, 2017; Published: January 12, 2018

\begin{abstract}
The binding of cefonicid sodium (CFS) with trypsin was investigated by spectroscopic and molecular docking methods under different temperatures conditions $(303,310$ and $318 \mathrm{~K}$ ). The results demonstrated that the interaction between CFS and trypsin was taking place via static quenching with 1:1 binding ratio. The fluorescence datas were treated by using the double logarithmic equation, and the binding constants $K_{a}$ of the interaction of CFS-trypsin systems and the number of binding sites $n$ were obtained. The thermodynamic parameters of CFS-trypsin systems under different temperatures were obtained by the thermodynamic equation. The experimental data show that the interactions between them were mainly hydrophobic interaction and hydrogen bonding interaction, and with the molecular docking results are consistent.
\end{abstract}

Keywords: Cefonicid Sodium, Trypsin, Spectrometry, Interaction, Molecular Docking

\section{Introduction}

Cefonicid Sodium (CFS) is a semisynthetic second generation cephalosporin whose structure, antibacterial activity, and stability to $\beta$-lactamases have been described and researched extensively (the structure shown in Figure 1). A number of studies have documented its broad spectrum of antibacterial activity against aerobic, anaerobic gram-positive and gram-negative bacteria effectively [1]. CFS is suitable for the following infections caused by sensitive bacteria: lower respiratory tract infection, urinary tract infection, septicemia, skin and soft tissue infections, bone and joint infection and it also can be used in surgery to prevent infection.

Trypsin (E. C. 3.4.21.4), a serine protease, is the most abundant proteases in nature and plays an essential role in digestion and deconstruction of food proteins and other physiological processes including hemostasis, apoptosis, signal transduction, reproduction, and immune response [2]. The molecular weight of trypsin is 23,300 Dalton and it consists of 223 amino acid residues [3]. The individual chains are held together by six disulfide bridges. It is composed of two domains of nearly equal size, the major constituent of each domain beinga set of six anti-parallel strands of polypeptide chain tied together into a $\beta$-sheet unit by a network of H-bonds [4]. Trypsin has four amino acids (Trp51, Trp141, Trp215, and Trp237) that can be used as intrinsic fluorophores [5]. Due to its essential physiological function, it has often been chosen as target protein to study the structural effects of small molecules to trypsin and thereby the function $[6,7]$. At present, the molecular interactions between trypsin and many ligands have been investigated successfully in biochemistry domain $[8,9]$. However, the interaction between CFS and trypsin has not been investigated. In this paper, the interaction of CFS with trypsin under different temperature conditions by used fluorescence spectroscopy and molecular docking technology. Furthermore, the thermodynamic parameters of the reaction between CFS and trypsin were obtained by the Van't Hoff equation. This study is expected to provide important insight into the essence, potential toxicity between drugs and protein in real terms, and can also provide a 
useful clinical reference for future combination therapy.<smiles>[NH3+]OC(=O)C1=C(CSc2nnnn2CS(=O)(=O)O[Na])CS[C@@H]2C(NC(=O)C(O)c3ccccc3)C(=O)N12</smiles>

Figure 1. Chemical structure of Cefonicid Sodium.

\section{Experimental}

\subsection{Apparatus}

All fluorescence spectra were recorded with a Shimadzu RF-5301PC spectrofluorophotometer. Absorption was measured with an UV-vis recording spectrophotometer (UV-265, Shimadzu, Japan). All pH measurements were carried out with a pHS-3C precision acidity meter (Leici, Shanghai, China). All temperatures were controlled by a SYC-15 B superheated water bath (Nanjing Sangli Electronic Equipment Factory).

\subsection{Materials}

Trypsin was purchased from Sigma Co. and was of the purity grade inferior $99 \%$. Cefonicid Sodium (CFS) as a standard in guarantee reagent, and was of the purity grade inferior $99 \%$. Stock solutions of trypsin $\left(4.0 \times 10^{-5} \mathrm{M}\right)$ and CFS $\left(1.0 \times 10^{-3} \mathrm{M}\right)$ were prepared. All the stock solutions were further diluted for use as working solutions. Tris- $\mathrm{HCl}$ buffer solution containing $0.15 \mathrm{M} \mathrm{NaCl}$ was used to maintain the $\mathrm{pH}$ of solutions at 7.40 and $\mathrm{NaCl}$ solution was used to maintain the ionic strength of the solution. All other reagents were of analytical grade and all aqueous solutions were prepared with newly double-distilled water and stored in dark at $4^{\circ} \mathrm{C}$.

The fluorescence intensities were corrected for the absorption of excitation light and re-absorption of emitted light to decrease the inner filter using the following relationship [10]:

$$
F_{c o r}=F_{o b s} \times e^{\left(A_{e x}+A_{e m}\right) / 2}
$$

Where, $F_{\text {cor }}$ and $F_{\text {obs }}$ are the corrected and observed fluorescence intensities, respectively. $A_{\mathrm{ex}}$ and $A_{\mathrm{em}}$ are the absorbance values of CFS at excitation and emission wavelengths, respectively. The fluorescence intensity used in this paper was corrected.

\subsection{Procedures}

\subsubsection{Fluorescence Measurements}

In a typical fluorescence measurement, $0.5 \mathrm{~mL}$ Tris- $\mathrm{HCl}$ $(\mathrm{pH}=7.40), 0.5 \mathrm{~mL}$ trypsin solution $\left(4.0 \times 10^{-5} \mathrm{M}\right)$, and different concentrations of cefonicid sodium were added into a $10 \mathrm{~mL}$ colorimetric tube successively. The samples were diluted to $5.0 \mathrm{~mL}$ with double-distilled water, mixed thoroughly by shaking, and kept static for $30 \mathrm{~min}$ at different temperatures $(303,310$ and $318 \mathrm{~K})$. The excitation wavelength for trypsin was $280 \mathrm{~nm}$ and $295 \mathrm{~nm}$, respectively, with a 1.0 $\mathrm{cm}$ quartz sampling cells. The excitation and emission slits were set at $5 \mathrm{~nm}$. The solution was subsequently scanned on the fluorophotometer and the fluorescent intensity of the system was recorded.

\subsubsection{Synchronous Fluorescence Measurements}

Preparation of solution is the same as that of the former. The fluorescence spectra of the CFS-trypsin systems were recorded when the $\Delta \lambda$ value between the excitation and emission wavelengths was stabilized at 15 and $60 \mathrm{~nm}$, respectively.

\subsubsection{UV-vis Absorption Measurements}

A volume of $1.0 \mathrm{~mL}$ of Tris- $\mathrm{HCl}(\mathrm{pH}=7.40), 1.0 \mathrm{~mL}$ trypsin solution $\left(4.0 \times 10^{-5} \mathrm{M}\right)$, and different concentrations of cefonicid sodium were added into $10 \mathrm{~mL}$ colorimetric tube successively. The samples were diluted to scaled volume with double-distilled water, mixed thoroughly by shaking, and kept static for $30 \mathrm{~min}$ at $303 \mathrm{~K}$. With appropriate concentration of cefonicid sodium as the blank reference, the ultraviolet absorbance of the determination of solution, and draw the ultraviolet absorbance of the CFS-trypsin systems spectrogram.

\subsubsection{Molecular Docking Investigation}

The crystal structure of trypsin (PDB ID:2PTN) was obtained from the Protein Data Bank, Structural formula of the CFS in Chemdraw Pro 14.0 software, At the same time in the Chembio 3D Ultra 14.0 software to optimize the energy of its three-dimensional structure. Finally use Autodock 4.2.6 software to molecular docking of CFS and trypsin, application of Lamarck (LGA) genetic algorithm to calculate the combined with protein drug molecules possible conformation [11].

\section{Results and Discussion}

\subsection{Ultraviolet Absorption Spectrum of CFS-Trypsin Systems}

It is well-known that UV-visible absorption measurement is a very simple method and applicable to explore the structural change and formation of a complex [12]. The absorption spectra of CFS-trypsin combinations are shown in Figure 2. Trypsin has two main absorption bands. The strong absorption peak at $210 \mathrm{~nm}$ is resulted from the $\pi \rightarrow \pi^{*}$ electronic transition of peptide backbone structure $\mathrm{C}=\mathrm{O}$, which can reflect the framework conformation of trypsin [13]. Another the weak absorbance of trypsin around $280 \mathrm{~nm}$ is primarily caused by tryptophan (Trp) and tyrosine (Tyr) (a very small extent on the amount of phenylalanine (Phe) and disulfide bonds) [14]. When CFS is gradually added, the intensity of the absorption peak at about $210 \mathrm{~nm}$ decreased and underwent an obviously red shift. The results suggest that the interaction between CFS and trypsin leads to loosen or destruct of the protein backbone 
$[15]$.

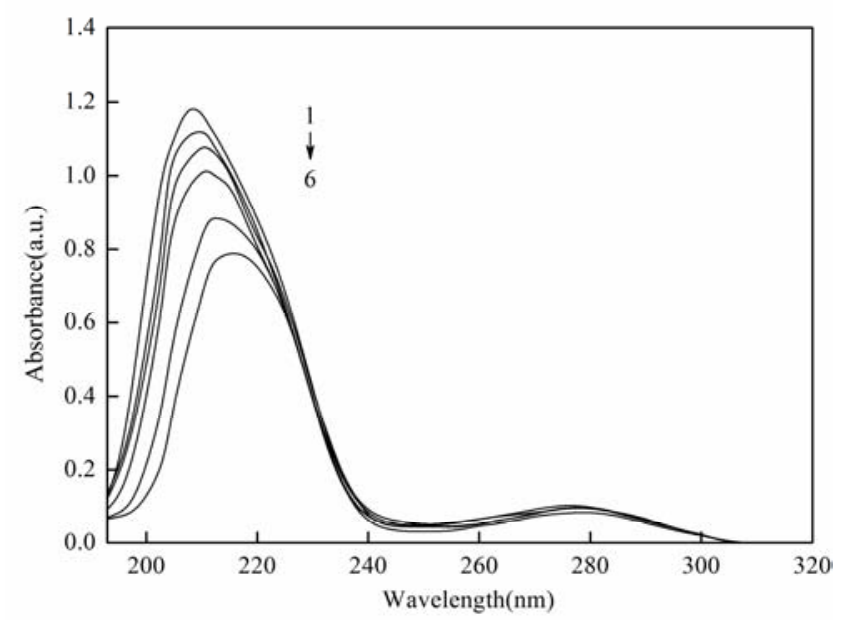

$\mathrm{C}_{\text {Trypsin }}=4.0 \times 10^{-6} \mathrm{~mol} / \mathrm{L}, 1 \sim 6 \mathrm{C}_{C F S}=(0,0.4,0.82 .0,4.0,5.0) \times 10^{-5} \mathrm{~mol} / \mathrm{L}$

Figure 2. Absorption spectrum of CFS-trypsin systems ( $T=298 \mathrm{~K})$.

\subsection{Fluorescence Spectra of CFS-Trypsin Systems}

Proteins are considered to have intrinsic fluorescence due to the presence of amino acids, mainly Trp, Tyr. When the excitation wavelengths were at $280 \mathrm{~nm}$ and $295 \mathrm{~nm}$, the emission peaks for trypsin were both located at $340 \mathrm{~nm}$. We recorded the fluorescence spectra of CFS-trypsin systems which were shown in Figure 3. As depicted in Figure 3, the fluorescence intensity of trypsin decreased regularly with the addition of CFS when the excitation wavelength was $280 \mathrm{~nm}$, which indicated that CFS could quench the intrinsic fluorescence of trypsin strongly and there was an interaction between CFS and trypsin.

In order to confirm the quenching mechanism, the fluorescence quenching data are analyzed by the Stern-Volmer equation [16] (2):

$$
F_{0} / F=1+K_{q} \tau_{0}[Q]=1+K_{s v}[Q]
$$

Where $F_{0}$ is the fluorescence intensities in the absence of CFS, $F$ is that in the presence of different concentration of CFS. $\tau_{0}$ is the average life time of the protein without the quencher $\left(10^{-8} \mathrm{~s}\right)$, and $[Q]$ is the concentration of the quencher. $K_{s v}$ and $K_{q}$ are the Stern-Volmer quenching constant and the quenching rate constant of biomolecular, respectively. Based on the linear fit plot of $F_{0} / F$ versus $[L]$, values of $K_{s v}$ and $K_{q}$ could be obtained at different temperatures. The calculated results were shown in Table 1. Table 1 showed that the value of $K_{s v}$ gradually decreased with rising temperature. That is to say, the extent of fluorescence quenching of CFS to trypsin was reduced with rising temperature. The results indicated that fluorescence quenching was not caused by diffusion and collision of dynamic quenching, but was static quenching process forming ground-state complex between CFS and trypsin [17]. In addition, values of $K_{q}$ were much greater than the maximum scatter collision quenching constant of various quenchers $\left(2 \times 10^{10} \mathrm{M}^{-1} \cdot \mathrm{s}^{-1}\right)$ under different temperatures, which also suggested that the quenching was a static process [18].

The binding constant $\left(K_{a}\right)$ and the number of binding sites (n) can be calculated by the double logarithm regression curve, shown in the following equation [19]:

$$
\log \left(\frac{F_{0}-F}{F}\right)=n \log K_{a}+\mathrm{n} \log \left\{\left[D_{t}\right]-n \frac{F_{0}-F}{F}\left[B_{t}\right]\right\}
$$

Where $\left[D_{t}\right]$ and $\left[B_{t}\right]$ are the total concentrations of CFS and trypsin, respectively. On the assumption that $n$ in the bracket is equal to 1 (the results remain the same no matter what the value of $n$ is hypothesized in the bracket, for example, $n=1,2$, $3,7.3$ or 13.7, the calculation remain the same). The curve of $\log \left(F_{0} / F-1\right)$ versus $\log \left\{\left[D_{t}\right]-\left[B_{t}\right]\left(1-F / F_{0}\right)\right\}$ is drawn and fitted linearly, then the value of $n$ can be obtained from the slope of the plot. If the $n$ value obtained is not equal to 1 , then it is substituted into the bracket and the curve of $\log \left(F_{0} / F-1\right)$ versus log $\left\{\left[D_{t}\right]-n\left[B_{t}\right]\left(F_{0}-F\right) / F_{0}\right\}$ is drawn again. The above process is repeated again and again till $n$ obtained is only a single value or a circulating value. The value of $n$ and binding constant $K_{a}$ can be obtained from the slope and the intercept of the plot which were shown in Table 1 . As seen in Table 1, values of $n$ were all about 1 , which suggested that just one binding site for CFS existed in trypsin. Meanwhile, the binding constants $K_{a}$ decreased with increasing temperature, that is to say, the stability of ground-state complex was reduced with rising temperature, further suggesting that the quenching was a static process [20].

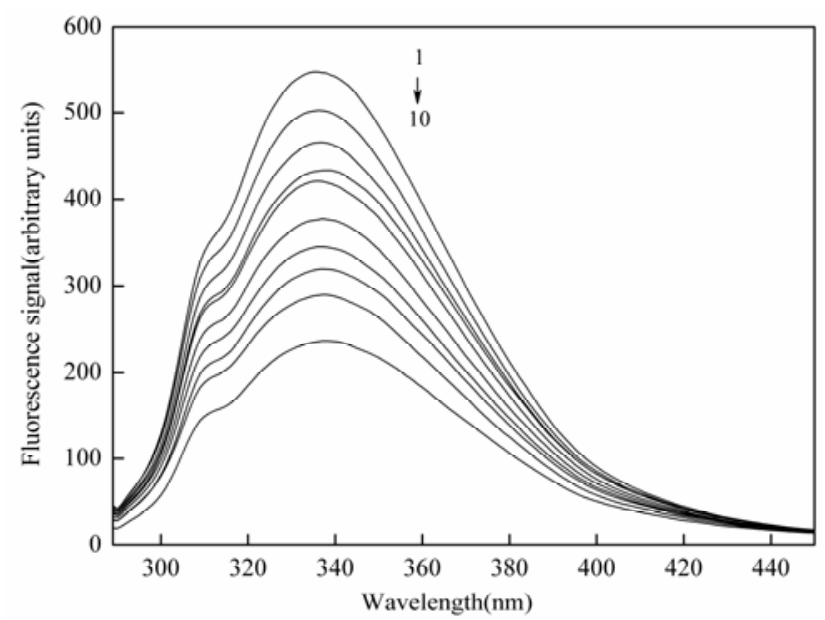

Figure 3. Fluorescence spectrum of CFS-trypsin systems $\left(T=303 \mathrm{~K}, \lambda_{e x}=\right.$ $280 \mathrm{~nm})$.

$C_{\text {Trypsin }}=4.0 \times 10^{-6} \mathrm{~mol} / \mathrm{L} ; 1 \sim 10 C_{C F S}=(0,0.4,0.6,1.0,2.0,3.0,4.0,5.0,6.0$, 8.0) $\times 10^{-5} \mathrm{~mol} / \mathrm{L}$ 
Table 1. Quenching reactive parameters of CFS and trypsin at different temperatures.

\begin{tabular}{llllllll}
\hline$\lambda_{e x} /(\mathbf{n m})$ & $\boldsymbol{T} /(\mathbf{K})$ & $\boldsymbol{K}_{q} /(\mathbf{L} / \mathbf{m o l} \cdot \mathbf{s})$ & $\boldsymbol{K}_{\text {sv }} /(\mathbf{L} / \mathbf{m o l})$ & $\boldsymbol{r}_{\mathbf{1}}$ & $\boldsymbol{K}_{\boldsymbol{a}} /(\mathbf{L} / \mathbf{m o l})$ & $\boldsymbol{n}$ & $\boldsymbol{r}_{\mathbf{2}}$ \\
\hline \multirow{2}{*}{280} & 303 & $1.65 \times 10^{12}$ & $1.65 \times 10^{4}$ & 0.9981 & $1.73 \times 10^{4}$ & 1.08 & 0.9983 \\
& 310 & $1.62 \times 10^{12}$ & $1.62 \times 10^{4}$ & 0.9950 & $1.65 \times 10^{4}$ & 0.99 & 0.9949 \\
& 318 & $1.48 \times 10^{12}$ & $1.48 \times 10^{4}$ & 0.9981 & $1.53 \times 10^{4}$ & 0.83 & 0.9981 \\
295 & 303 & $9.23 \times 10^{11}$ & $9.23 \times 10^{3}$ & 0.9904 & $9.34 \times 10^{3}$ & 0.96 & 0.9977 \\
& 310 & $8.32 \times 10^{11}$ & $8.32 \times 10^{3}$ & 0.9949 & $8.43 \times 10^{3}$ & 0.89 & 0.9984 \\
& 318 & $7.56 \times 10^{11}$ & $7.56 \times 10^{3}$ & 0.9925 & $7.82 \times 10^{3}$ & 0.85 & 0.9931 \\
\hline
\end{tabular}

$r_{l}$ is the linear relative coefficient of $F_{0} / F \sim[Q] ; r_{2}$ is the linear relative coefficient of $\log \left(F_{0}-F\right) / F \sim \log \left\{\left[D_{\mathrm{t}}\right]-n\left[B_{\mathrm{t}}\right]\left(F_{0}-F\right) / F_{0}\right\}$.

\subsection{The Participation of Amino Acid Residue Studies in CFS-Trypsin Systems}

At $280 \mathrm{~nm}$ wavelength, the Try and Tyr residues in trypsin are excited, whereas the $295 \mathrm{~nm}$ wavelength excites only tryptophan residues [21]. Based on the Stern-Volmer equation, comparing the fluorescence quenching of protein excited at $280 \mathrm{~nm}$ and $295 \mathrm{~nm}$ allows to estimate the participation of Trp and Tyr groups in CFS-trypsin systems [22]. From Figure 4, in the presence of CFS, the quenching curves of trypsin excited at $280 \mathrm{~nm}$ and $295 \mathrm{~nm}$ did not overlap, and the extent of fluorescence quenching at $280 \mathrm{~nm}$ was larger than the extent of fluorescence quenching at $295 \mathrm{~nm}$. This showed that tryptophan and tyrosine residues both participated in the CFS-trypsin complexation process. From Table 1, at the same temperature, the values of $K_{a}$ at excitation wavelengths of 280 $\mathrm{nm}$ and $295 \mathrm{~nm}$ showed differences, which also shown that tryptophan and tyrosine residues both participated in the interaction between CFS and trypsin.

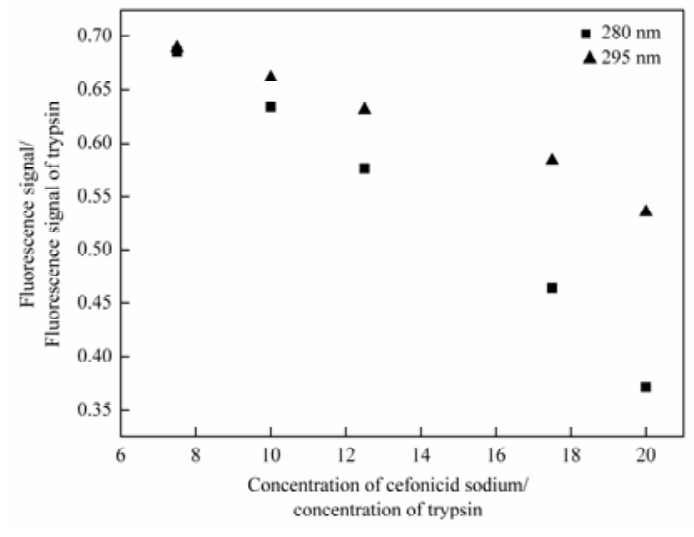

$C_{\text {Trypsin }}=4.0 \times 10^{-6} \mathrm{~mol} / \mathrm{L} ; C_{\mathrm{CFS}}=(0.4,0.6,1.0,2.0,3.0) \times 10^{-5} \mathrm{~mol} / \mathrm{L}$

Figure 4. Quenching curves of CFS-trypsin systems $\left(\lambda_{e x}=280\right.$ and $\left.295 \mathrm{~nm}\right)$.

\subsection{Synchronous Fluorescence Spectra of CFS-Trypsin Systems}

When the value of $\Delta \lambda$ between the excitation and emission wavelengths is stabilized at either $15 \mathrm{~nm}$ or $60 \mathrm{~nm}$, synchronous fluorescence gives characteristic information for Tyr or Trp, respectively [23]. It can be seen from Figure 5, the fluorescence intensity of both Try and Tyr residues decreased regularly, but the emission wavelength of the Try residues was red shifted with increasing concentration of CFS. At the same time, there was no change in the emission wavelength of Tyr residues. The red shift effect suggested that the microenvironment of around Try residues was changed and the hydrophility of tryptophan residues increased in the presence of CFS [24]. According to the equation (2) and (3), the quenching parameters for $\Delta \lambda=60 \mathrm{~nm}$ and $\Delta \lambda=15 \mathrm{~nm}$ are shown in Table 2. From Table 2, it can be seen that the values of $K_{\mathrm{sv}}$ decreased with increasing temperatures for CFS-trypsin systems, which indicated that the probable quenching mechanism of the interaction between CFS and trypsin was a static process. At the same time, the values of $K_{q}$ were much greater than the maximum scatter collision quenching constant of various quenchers $\left(2 \times 10^{10} \mathrm{M}^{-1} \cdot \mathrm{s}^{-1}\right)$ under different temperatures, which proved that the quenching process was a static quenching process with the formation of a new complex [25]. The $n$ value approaches unity, suggesting that CFS binds with trypsin moderately through a 1:1 binding mode; the decreasing trend of the Ka with increasing temperature was conformed with static quenching process [26].
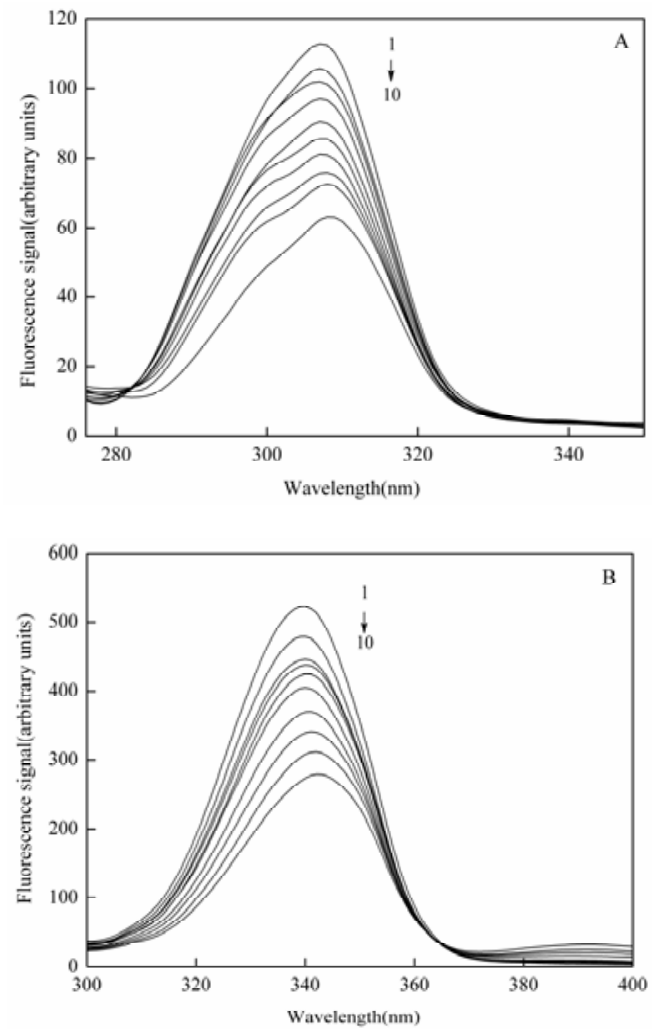

Figure 5. Fluorescence spectrum of CFS-trypsin systems $(T=303 \mathrm{~K})(\mathrm{A})$ $\Delta \lambda=15 \mathrm{~nm} ;(B) \Delta \lambda=60 \mathrm{~nm}$.

$\mathrm{C}_{\text {Trypsin }}=4.0 \times 10^{-6} \mathrm{~mol} / \mathrm{L} ; 1 \sim 10 \mathrm{C}_{C F S}=(0,0.4,0.6,1.0,2.0,3.0,4.0,5.0,6.0$, $8.0) \times 10^{-5} \mathrm{~mol} / \mathrm{L}$ 
Table 2. Quenching reactive parameters of CFS and trypsin at different temperatures.

\begin{tabular}{llllllll}
\hline $\boldsymbol{\Delta} \lambda /(\mathbf{n m})$ & $\boldsymbol{T} /(\mathbf{K})$ & $\boldsymbol{K}_{q} /(\mathbf{L} / \mathbf{m o l} \cdot \mathbf{s})$ & $\boldsymbol{K}_{s v} /(\mathbf{L} / \mathbf{m o l})$ & $\boldsymbol{r}_{1}$ & $\boldsymbol{K}_{a} /(\mathbf{L} / \mathbf{m o l})$ & $\boldsymbol{n}$ & $\boldsymbol{r}_{\mathbf{2}}$ \\
\hline \multirow{3}{*}{15} & 303 & $1.00 \times 10^{12}$ & $1.00 \times 10^{4}$ & 0.9945 & $1.10 \times 10^{4}$ & 0.97 & 0.9903 \\
& 310 & $9.88 \times 10^{11}$ & $9.88 \times 10^{3}$ & 0.9980 & $9.67 \times 10^{3}$ & 0.87 & 0.9967 \\
& 318 & $9.84 \times 10^{11}$ & $9.84 \times 10^{3}$ & 0.998 & $9.15 \times 10^{3}$ & 0.81 & 0.9937 \\
60 & 303 & $1.39 \times 10^{12}$ & $1.39 \times 10^{4}$ & 0.9945 & $1.48 \times 10^{4}$ & 0.99 & 0.9975 \\
& 310 & $1.32 \times 10^{12}$ & $1.32 \times 10^{4}$ & 0.9919 & $1.31 \times 10^{4}$ & 0.81 & 0.9928 \\
& 318 & $1.14 \times 10^{12}$ & $1.14 \times 10^{4}$ & 0.9956 & $1.29 \times 10^{4}$ & 0.83 & 0.9975 \\
\hline
\end{tabular}

$K_{q}$ quenching rate constant; $K_{a}$ is the binding constant; $n$ is the number of binding site; $r_{3}$ is the linear relative coefficient of $F_{0} / F \sim[\mathrm{L}] ; r_{4}$ is the linear relative coefficient of $\log \left(F_{0}-F\right) / F \sim \log \left\{\left[\mathrm{D}_{\mathrm{t}}\right]-\mathrm{n}\left[\mathrm{B}_{\mathrm{t}}\right]\left(F_{0}-F\right) / F_{0}\right\}$.

\subsection{Type of Interaction Force of CFS-Trypsin Systems}

In general, the interaction between biological macromolecules and small molecules can be described by four types of interaction: hydrophobic, hydrogen bonding, van der Waals and electrostatic interactions [27]. The type of interaction force of CFS-trypsin systems can be gained through the thermodynamic parameters of reaction of CFS with trypsin. Some thermodynamic parameters like standard enthalpy change $(\Delta H)$, standard entropy change $(\Delta S)$ and Gibbs free energy change $(\Delta G)$ of binding reaction are the functions of temperature and can be regarded as the basic criteria confirming the binding model [28]. Therefore, these parameters were calculated from the van't Hoff equation: [29]

$$
\begin{gathered}
R \ln K_{a}=-\Delta H / T+\Delta S \\
\Delta G=\Delta H-T \Delta S
\end{gathered}
$$

Where $R$ is the gas constant $(\mathrm{R}=8.314 \mathrm{~mol} \cdot \mathrm{K} / \mathrm{J}), T$ is the experimental temperature and $K_{a}$ is binding constant at corresponding temperature. According to the thermodynamic parameters of small molecules and biological macromolecules, the interaction types can be easily judged. For CFS-trypsin systems, based on the $K_{a}$ value of different temperatures, the calculated results were shown in Table 3 . The negative value of $\Delta G$ clarified a spontaneous reaction between CFS and trypsin. The negative value of $\Delta H$ and positive value of $\Delta S$ showed that the reaction between CFS and trypsin mainly electrostatic attraction [30]. Furthermore, synchronous fluorescence and fluorescence quenching two methods got the same conclusion.

Table 3. The thermodynamic parameters of CFS-trypsin at different

\begin{tabular}{|c|c|c|c|c|c|}
\hline & $T /(\mathbf{K})$ & $\begin{array}{l}K_{a} / \\
(\mathrm{L} / \mathrm{mol})\end{array}$ & $\begin{array}{l}\Delta H / \\
(\mathrm{KJ} / \mathrm{mol})\end{array}$ & $\begin{array}{l}\Delta S / \\
(\mathrm{J} / \mathrm{mol} \cdot \mathrm{K})\end{array}$ & $\begin{array}{l}\Delta G \\
/(\mathrm{KJ} / \mathrm{mol})\end{array}$ \\
\hline \multirow{3}{*}{$\begin{array}{l}\Delta \lambda=15 \mathrm{n} \\
\mathrm{m}\end{array}$} & 303 & $1.10 \times 10^{4}$ & \multirow{3}{*}{-9.77} & 45.12 & -23.44 \\
\hline & 310 & $9.67 \times 10^{3}$ & & 44.77 & -23.65 \\
\hline & 318 & $9.15 \times 10^{3}$ & & 45.11 & -24.11 \\
\hline \multirow{3}{*}{$\begin{array}{l}\Delta \lambda=60 \mathrm{n} \\
\mathrm{m}\end{array}$} & 303 & $1.48 \times 10^{4}$ & \multirow{3}{*}{-7.25} & 55.90 & -24.19 \\
\hline & 310 & $1.31 \times 10^{4}$ & & 55.43 & -24.43 \\
\hline & 318 & $1.29 \times 10^{4}$ & & 55.89 & -25.02 \\
\hline \multirow{3}{*}{$\begin{array}{l}\lambda_{e x}= \\
280 \mathrm{~nm}\end{array}$} & 303 & $1.73 \times 10^{4}$ & \multirow{3}{*}{-6.57} & 59.42 & -24.59 \\
\hline & 310 & $1.65 \times 10^{4}$ & & 59.52 & -25.02 \\
\hline & 318 & $1.53 \times 10^{4}$ & & 59.42 & -25.47 \\
\hline \multirow{3}{*}{$\begin{array}{l}\lambda_{e x}= \\
295 \mathrm{~nm}\end{array}$} & 303 & $9.43 \times 10^{3}$ & \multirow{3}{*}{-9.46} & 44.79 & -23.03 \\
\hline & 310 & $8.43 \times 10^{3}$ & & 44.64 & -23.29 \\
\hline & 318 & $7.82 \times 10^{3}$ & & 44.79 & -23.70 \\
\hline
\end{tabular}
temperatures in two ways.

\subsection{Hill's Coefficient of CFS-Trypsin Systems}

According to the Hill's coefficient of CFS-trypsin systems, we can make a quantitative analysis for cooperative binding between protein and ligands on the basis of the following equation: [31]

$$
\lg \frac{Y}{1-Y}=\lg K+n_{H} \lg [L]
$$

Where $K_{a}$ is the binding constant, $Y$ is the fractional binding saturation, and $n_{H}$ is the Hill's coefficient. Hill's coefficient is greater than 1, which exhibits positive cooper-activity and its role is enhanced with increasing $n_{H}$. Conversely, Hill's coefficient is less than 1 , which exhibits negative cooper-activity and its role is enhanced by decreasing $n_{H}$. A coefficient of 1 indicates non-cooperative reaction.

For fluorescence measurement:

$$
\begin{aligned}
& \frac{Y}{1-Y}=\frac{Q}{Q_{m}-Q} \\
& Q=\frac{F_{0}-F}{F_{0}}
\end{aligned}
$$

Where $1 / Q_{m}$ is intercept of the plot $1 / Q$ versus $1 /[L]$. According to the formula (6), Hill's coefficient of CFS-trypsin systems can be gained from the slope of the plot of $\log [Y /(1-Y)]$ versus $\log [L]$. The results were presented in Table 4 . We could see that the values of $n_{H}$ were slightly greater than 1 at different temperatures, which indicated that there were positive cooperative reaction between trypsin and CFS. Meanwhile, it illustrated that with the increase of temperature, the concentration of CFS on the fluorescence quenching of trypsin was more difficulties, and the binding constant decreases with the increase of the temperature. Finally, the experimental results show that the synergy between the fluorescence quenching method and the synchronous fluorescence method is consistent with the CFS-trypsin systems.

Table 4. Hill coefficient of CFS-trypsin systems at different temperatures.

\begin{tabular}{lllllllll}
\hline \multirow{2}{*}{$\mathbf{T} / \mathbf{K}$} & \multicolumn{2}{c}{$\boldsymbol{\lambda} \mathbf{e x}=\mathbf{2 8 0} \mathbf{~ n m}$} & \multicolumn{2}{c}{$\boldsymbol{\lambda} \mathbf{e x}=\mathbf{2 9 5} \mathbf{~ n m}$} & \multicolumn{2}{c}{$\boldsymbol{\Delta} \boldsymbol{\lambda}=\mathbf{1 5} \mathbf{~ n m}$} & \multicolumn{2}{c}{$\boldsymbol{\Delta} \boldsymbol{\lambda}=\mathbf{6 0} \mathbf{~ n m}$} \\
\cline { 2 - 8 } & $\boldsymbol{n}_{\boldsymbol{H}}$ & $\mathbf{r}_{\mathbf{3}}$ & $\boldsymbol{n}_{\boldsymbol{H}}$ & $\mathbf{r}_{\mathbf{3}}$ & $\boldsymbol{n}_{\boldsymbol{H}}$ & $\mathbf{r}_{\mathbf{3}}$ & $\boldsymbol{n}_{\boldsymbol{H}}$ & $\mathbf{r}_{\mathbf{3}}$ \\
\hline 303 & 0.94 & 0.9902 & 1.02 & 0.9981 & 1.02 & 0.9920 & 1.22 & 0.9906 \\
310 & 0.97 & 0.9952 & 0.97 & 0.9989 & 1.00 & 0.9956 & 1.12 & 0.9917 \\
318 & 1.02 & 0.9941 & 1.01 & 0.9992 & 1.04 & 0.9901 & 1.24 & 0.9904 \\
\hline
\end{tabular}

$r_{3}$ is the linear relative coefficient of $\log [\mathrm{Y} /(1-\mathrm{Y})] \sim \log [\mathrm{L}], n_{H}$ is the Hill's coefficient. 


\subsection{Binding Distances between Trypsin and CFS}

According to Förster's non-radiative energy transfer theory [32], the distance $(r)$ between the donor and the acceptor, critical energy transfer distance $R_{0}$ when the transfer efficiency is $50 \%$ and the energy-transfer efficiency $E$ can be calculated by the formulas: [33]

$$
\begin{gathered}
E=\frac{R_{0}{ }^{6}}{R_{0}{ }^{6}+r^{6}}=1-\frac{F}{F_{0}} \\
R_{0}{ }^{6}=8.78 \times 10^{-25} K^{2} \Phi N^{-4} J \\
J=\frac{\sum F(\lambda) \varepsilon(\lambda) \lambda^{4} \Delta \lambda}{\sum F(\lambda) \Delta \lambda}
\end{gathered}
$$

Where $F_{0}$ is the fluorescence intensity of donor, $F$ is the fluorescence intensity for the donor in presence of same concentration acceptor, $K^{2}$ is the orientation factor, $\Phi$ is the fluorescence quantum yield of the donor in the absence of acceptor, $N$ is a refractive index of the medium, $F(\lambda)$ is the fluorescence intensity of the fluorescence donor at wavelength $\lambda$ and $\varepsilon(\lambda)$ is the molar absorption coefficient of the acceptor at this wavelength. Under these experimental conditions, it has been reported that $K^{2}=2 / 3, N=1.336$ and $\Phi=0.118$ [34]. $J$ is the overlap integral between the fluorescence emission spectrum of the donor and the absorption spectrum of the acceptor. Thus $J, E, R_{0}$ and $r$ were calculated and shown in Table 5. As seen in Table 5, the donor-to-acceptor distance $r<7 \mathrm{~nm}$ indicated that the energy transfer from trypsin to CFS occurred with high possibility [35]. The distance $r$ increased and the energy efficiency $E$ decreased with increasing temperature, which resulted in the reduced stability of the binary systems and the values of $K_{a}$. Moreover, the value of $r$ was greater than $R_{0}$ in this study which suggested that CFS could strongly quench the intrinsic fluorescence of trypsin by a static quenching mechanism.

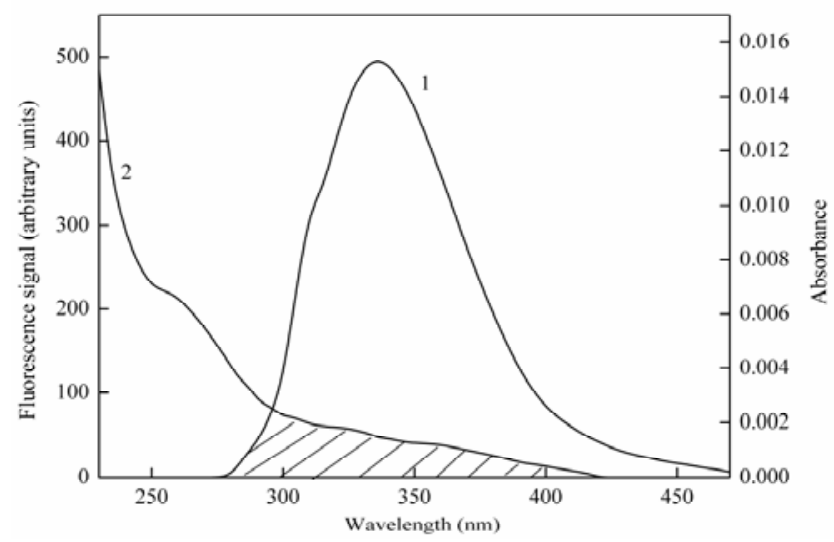

Figure 6. Overlap of the fluorescence spectrum of trypsin $\left(\lambda_{e x}=280 \mathrm{~nm}\right)$ (1) and the absorption spectrum of CFS (2) $(T=303 K), C_{C F S}=C_{\text {Trypsin }}$ $=4.0 \times 10^{-6} \mathrm{~mol} / \mathrm{L}$.
Table 5. Binding parameters between trypsin and CFS at different temperatures.

\begin{tabular}{lllll}
\hline $\boldsymbol{T} /(\mathbf{K})$ & $\boldsymbol{E} /(\boldsymbol{\%})$ & $\boldsymbol{J} /\left(\mathbf{c m}^{3} \cdot \mathbf{L} / \mathbf{m o l}\right)$ & $\boldsymbol{R}_{\boldsymbol{0}} /(\mathbf{n m})$ & $\boldsymbol{r} /(\mathbf{n m})$ \\
\hline 303 & 3.94 & $6.03 \times 10^{-16}$ & 1.53 & 2.61 \\
310 & 3.86 & $6.15 \times 10^{-16}$ & 1.62 & 2.74 \\
318 & 3.83 & $6.23 \times 10^{-16}$ & 1.59 & 2.72 \\
\hline
\end{tabular}

\subsection{Molecular Docking}

Molecular simulation technology was widely used in the analysis of the interaction between protein and ligand. The aim of molecular docking was finded the best binding position between the substrate and the receptor molecule. The optimal binding position of trypsin and drug small molecule was selected by molecular docking software, the docking results data processing shows that, the combination of CFS and trypsin for $25.05 \mathrm{KJ} / \mathrm{mol}$, and the results with the experiment of the proceeds of the thermodynamic parameters are very close to $(\Delta G=25.02 \mathrm{KJ} / \mathrm{mol})$, which is further evidence of the interaction between CFS and trypsin.

As shown in Figure 7, it is the best conformation of CFS with trypsin molecular docking. CFS molecules are ammonia acid residues Tyr59, Cys58, Lys60, Phe41, Cys42, Cys58, His57, Ser195, Gly193, Gln192, Cys191, Trp215, Gly216, Gly219 and Cys220 by surrounded, most amino acid residues of hydrophobic amino acid residues. This result indicates that the hydrophobic interaction exists between CFS and typsin, and the electrostatic attraction is the main force. And CFS can effectively quench the trypsin fluorescence, which is consistent with the experimental results of fluorescence spectra. Figure 7 is the best conformation of CFS and trypsin molecular docking.

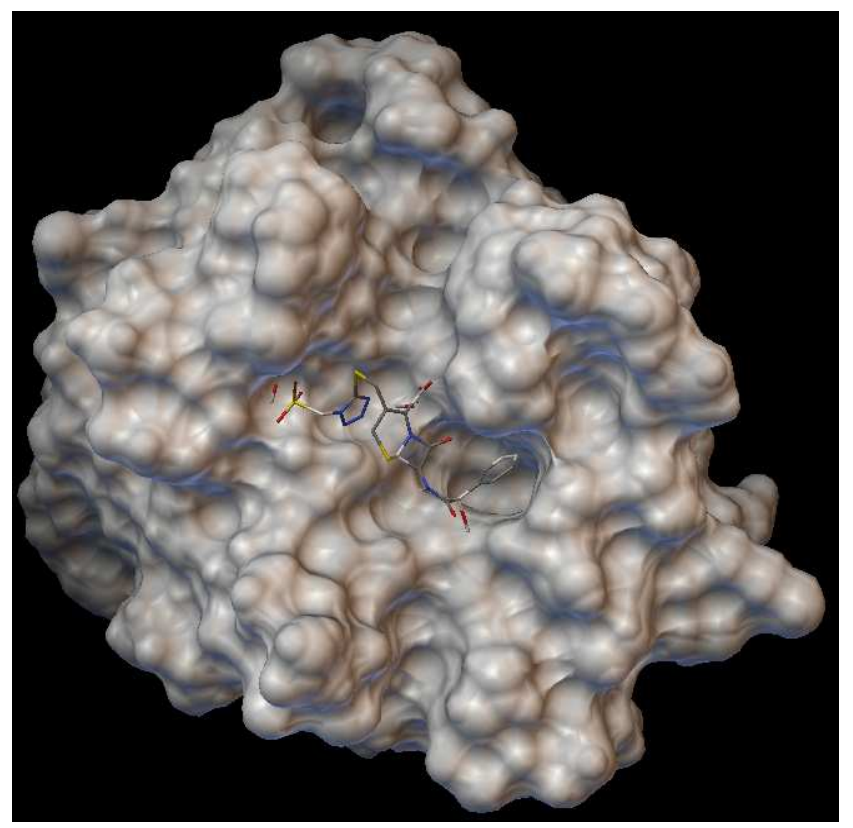

(1) 


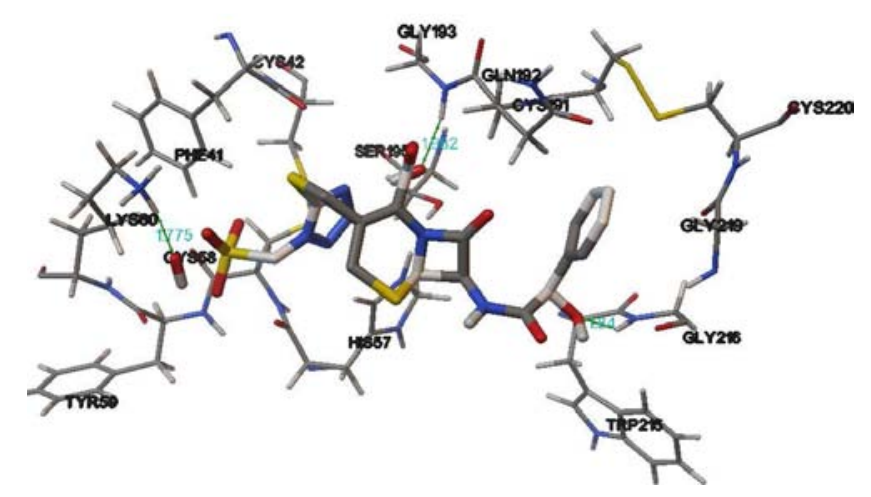

(2)

Figure 7. Computation docking model of the interaction between CFS and trypsin. (1) Binding site in the trypsin cavity. (2) Detailed illustration of the amino acid residues lining the binding site in the trypsin cavity.

More clearly from the Figure 7 found that CFS with Lys60, Ser195 and Gly216 form hydrogen bond, bond length were $1.775,1.982,1.840 \AA$. The results show that there are strong hydrogen bonding effect between CFS and trypsin. Therefore, the research results show that the combination of molecular docking model of CFS and trypsin is mainly the hydrophobic interaction, hydrogen bonding interactions exist at the same time, consistent with the experimental results.

\section{Conclusions}

In this work, the interaction of trypsin with CFS has been studied by using spectroscopic techniques and molecular docking method. The thermodynamic parameters indicated that the interaction between CFS and trypsin was spontaneous and hydrophobic forces played primary roles in stabilizing the CFS-trypsin complex. Besides, the molecular docking results showed the specific binding site and the detailed information of hydrogen bond, and hydrophobic interaction between the protein and ligand. At the same time, the performed results provide some important information on the structure and function of protein and may be useful for pharmacology and biochemistry.

\section{Acknowledgments}

The authors gratefully acknowledge financial support of the National Science Foundation of China (Grant No. 20675024) and the Hebei Provincial Key Basic Research Program (Grant No.10967126D).

\section{References}

[1] X. N. Yan, B. S. Liu, B. H. Chong, S. N. Cao, J. Lumin. 2013, 142,162 .

[2] L. Gombos, J. Kardos, A. Patthy, P. Medveczky, L. Szilagyi, A. Malnasi-Csizmadia, L. Graf, Biochem. 2008, 47, 1684.

[3] S. Ghosh, Colloid. Surface. B 2008, 66, 186.

[4] R. M. Stroud, L. M. Kay, R. E. Dickerson, J. Mol. Biol. 1974,
83, 192.

[5] H. Li, J. Pu, Y. Wang, C. Liu, J. Yu, T. Li, R. Q. Wang, Spectrochim. Acta A 2013, 115, 11.

[6] Y. Q. Wang, C. Y. Tan, S. L. Zhuang, P. Z. Zhai, Y. Cui, Q. H. Zhou, H. M. Zhang, Z. H. Fei, J. Hazard. Mater. 2014, 278, 65.

[7] X. X. Hu, Z. H. Yu, R. T. Liu, Spectrochim. Acta A 2013, 108, 54.

[8] X. R. Li, P. H. Li, Lumin. 2016, 31, 792.

[9] M. Nidhin, D. Ghosh, H. Yadav, N. Yadav, S. Majumder, Materi. Sci. Engin. B 2015, 202, 53.

[10] W. He, H. Dou, L. Zhang, L. Wang, R. Wang, J. Chang, Spectrochim. Acta Part A 2014,118, 519.

[11] G. Elmas, Y. Esra, J. Fluoresc. 2014, 24, 1445.

[12] J. Q. Liu, J. N. Tian, Y. Li, X. J. Yao, Z. D. Hu, X. G. Chen, Macromol. Biosci. 2005, 4, 520.

[13] S. H. D. P. Lacerda, J. J. Park, C. Meuse, D. Pristinski, M. L. Becker, A. Karim, J. F. Douglas, Acs Nano 2009, 4, 379.

[14] C. N. Pace, F. Vajdos, L. Fee, Fee, G. Grimsley, T. Gray, Prot. Sci. 1995, 11, 2423.

[15] Z. X. Chi, R. T. Liu, H. Zhang, Biomacromolecules 2010, 9, 2459.

[16] R. Yang, L. L. Yu, H. J. Zeng, R. L. Liang, X. L. Chen, L. B. Qu, J. Fluoresc. 2012, 22, 1459.

[17] Y. Teng, F. Ji, C. Li, Z. Yu, R. Liu, J. Lumin. 2011, 131, 2667.

[18] G. Kaur, S. Tripathi, Spectrochim. Acta. Part A 2015, 134, 183.

[19] Y. Q. Wang, H. M. Zhang, G. C. Zhang, S. X. Liu, Q. H. Zhou, Z. H. Fei, Int. J. Biol. Macromol. 2007, 41, 250.

[20] Y. Hu, S. Xu, X. Zhu, A. Gong, Spectrochim. Acta A. 2009, 74, 531.

[21] R. Ghosh, M. Mukherjee, K. Chattopadhyay, S. Ghosh, J. Phys. Chem. B 2012, 116, 12500.

[22] M. Maciazek-Jurczyk, A. Sulkowska, B. Bojko, J. Rownicka, W. W. Sulkowska, J. Mol. Struct. 2009, 924-926, 384.

[23] Y. J. Hu, Y. Liu, Z. B. Pi, S. S. Qu, Bioorg. Med. Chem. 2005, 13,6609 .

[24] Y. Y. Hu, S. Q. Xu, X. S. Zhu, A. Q. Gong, Spectrochim. Acta Part A 2009, 74, 531.

[25] K. Gurvir, S. K. Tripathi, Spectrochim. Acta Part A 2015, 134, 183.

[26] Y. Y. Yue, X. G. Chen, J. Qin, X. J. Yao, Dyes Pigm. 2008, 79, 182.

[27] P. D. Ross, S. Subramanian, Biochem. 1981, 20, 3096.

[28] W. R. Wang, R. R. Zhu, R. Xiao, H. Liu, S. L. Wang, Biol. Trace. Elem. Res., 2011, 142, 446.

[29] Y. Lu, G. K. Wang, X. M. Lu, J. Lv, M. H. Xu, W. W. Zhang, Spectrochim. Acta Part A 2010, 75, 266.

[30] B. Bojko, A. Sulkowska, M. Maciazek-Jurczyk, J. Rownicka, W. W. Sukowski, J. Pharm. Biomed. Anal. 2010, 52, 384. 
[31] S. N. Khan, B. Islam, R. Yennamalli, A. Sultan, N. Subbarao, A. U. Khan, Eur. J. Pharm. Sci. 2008, 35, 382.

[32] W. He, H. J. Dou, L. J. Wang, R. Y. Wang, J. B. Chang, Spectrochim. Acta A 2014, 118, 519.

[33] J. Jin, X. Zhang, J. Lumin. 2008, 128, 86.
[34] R. Zhang, T. Sun, C. G. Liu, W. Song, Z. Z. Cao, R. T. Liu, Biochem. Mol. Toxicol. 2015, 29, 425.

[35] Y. Jia, H. Q. Yang, L. Q. Guo, Q. Wang, Q. Y. M. Huang, H. Li, Chemi. Resea. Applic. 2016, 28, 680. 